\title{
Forschungsstand - old bottle, new wine?
}

In diesem Kapitel geht es darum, wie Future Skills definiert sind, was bestehende Future Skills Ansätze umfassen, wie der Forschungsstand zu Future Skills und zu wichtigen Begriffen und Konzepten aussieht. Sind Future Skills etwas Neues oder nur new wine in old bottles? „Old bottle, new wine“ ist der Titel eines Jazz Albums vom Komponisten, Arrangeur und Pianisten Gil Evans aus dem Jahr 1958, in dem er mit seiner Band berühmte Jazzstücke wie beispielsweise „Bird Feathers“ von Charlie Parker neu interpretiert. Der Titel spielt mit der Idee, Altbekanntes noch einmal in neuer Verpackung zu präsentieren. Auch bei dem Konzept der Future Skills stellt sich die Frage: Was ist eigentlich dran, an dem neuen, populären Begriff? Was ist der wirkliche Gehalt des Konzeptes und was ist daran neu?

\section{B 1.1 Zur Definition und zum Konzept von Future Skills}

\#Future Skills sind definiert als Kompetenzen, die es Individuen erlauben in hochemergenten Organisations- und Praxiskontexten selbstorganisiert (erfolgreich) handlungsfähig zu sein. Damit sind Future Skills im eigentliche Sinne Kompetenzen. Sie sind eingebettet in den Diskurs um das Ziel von Hochschulbildung und Employability als Ziel jeglichen Bildungsprozess, der auf Beruflichkeit jeglicher Art hinzielt. Grundsätzlich gibt es zwei sich herausbildende Verständnisse und Verwendungsweisen des Begriffes Future Skills: Eine additiv-anreicherungsorientierte, die Future Skills als Zusatzkomponenten für Bildungsprozesse versteht, mit denen die eigentlichen Wissensvermittlungsvorgänge angereichert werden müssten, damit Studierende für zukünftige Tätigkeitsbereiches gut qualifiziert sind. Diese Sichtweise betont etwa die Bedeutung von digitalen Kompetenzen oder auch sog. Soft-Skills wie Kommunikation oder Präsentationsfähigkeiten. In der Diskussion um die Bedeutung dieser Fähigkeiten als zum bestehenden Cur- 
riculum zusätzlich hinzuzufügende Fähigkeiten haben sich in den Hochschulen in den 90er Jahren des letzten Jahrhunderts viele Zentren und Programme für Schlüsselqualifikationen gebildet. Vertreter dieser Sichtweise auf Future Skills in Deutschland sind beispielsweise der Stifterverband der Deutschen Wissenschaft mit der Future Skills Initiative. ${ }^{18}$

$\mathrm{Zu}$ dieser Sichtweise tritt in den letzten zehn Jahre verstärkt eine zweite Sichtweise, die der ersten mittlerweile konkurrierend gegenübersteht. In dieser zweiten Sichtweise werden Future Skills in einer integrativen Weise aufgefasst. Dabei wird eher der Bildungsprozess als Ganzes in den Blick genommen. In diesem Verständnis zielt das Konzept der Future Skills darauf, Bildungsprozesse so zu gestalten, dass Wissensvermittlung und die Entwicklung von Skills nicht als zwei Additive, sondern als ein integratives Konzept gesehen wird. In dieser Sichtweise sind Bildungsprozesse so zu gestalten, dass in Hochschullehrveranstaltungen grundsätzlich die Entwicklung von Kompetenzen und nicht nur die Vermittlung von Wissen angestrebt werden soll. Vertreter dieser Sichtweise auf Future Skills sind, im Ausland die Universität Toronto mit einer kanadischen Future Skills Initiative ${ }^{19}$, das World Economic Forum (WEF) mit einer Initiative zu „Skills for the Future ${ }^{\text {"20 }}$ oder die Organisation for Economic Co-operation and Development (OECD) mit der Initiative Future of Education and Skills 2030 21 . Unterschieden wird hierbei oftmals noch in domänenspezifische Kompetenzen und generische oder domänenübergreifende Kompetenzen (siehe hierzu beispielsweise Villa Sánchez \& Poblete Ruiz 2008). Jedoch ist der Fokus dieser Sichtweise auf Future Skills die Handlung eines Individuums, bzw. eines zukünftigen Professionals. Handlung als Bezugspunkt integriert dabei immer Bestände von Wissen, Motivation, Willen, Haltung und Wertvorstellungen in ein komplexes Gefüge von Dispositionen, die dann in einer Handlung als Performanz Ausdruck finden können.

Das NextSkills Projekt nimmt seinen Ausgang im zweiten der beschriebenen Auffassungen von Future Skills. Future Skills werden aufgefasst als Handlungsdispositionen, die sich in komplexen und unvorbereiteten zukünftigen Handlungssituationen als (erfolgreiche) kompetente Handlung manifestiert.

Unabhängig von der Sichtweise findet das Konzept der Future Skills in Hochschulen eine ständig steigende Relevanz. Diese hat mit den bereits beschriebenen Faktoren zu tun, und zusätzlich damit, dass Berufsausbildungen sich weltweit zunehmend akademisieren und das Hochschulstudium daher in immer größerer

18 https://www.stifterverband.org/future-skills

19 https://futureskillscanada.com

20 https://www.weforum.org/focus/skills-for-your-future

21 https://www.oecd.org/education/2030-project/ 
Weise gefragt ist, Employability und Fähigkeiten für einen gestaltenden Umgang mit einer immer komplexer werdenden Umwelt zu vermitteln.

\section{B 1.2 Zum Begriffsumfeld des Future Skills Konzeptes}

Der Begriff Future Skills ist ein neuer Kunstbegriff, der so nicht im erziehungswissenschaftlichen oder organisationssoziologischen Umfeld verankert ist, weder in der Lernpsychologischen Forschung noch in der Managementlehre auftaucht. In den folgenden Abschnitten wollen wir ihn begrifflich konstituieren und anhand der Konzepte Bildung, Lernen, Kompetenz und Selbstorganisation bestimmen.

\section{B 1.2.1 Bildungsbegriffliche und lerntheoretische Einordnung von Future Skills}

Bildung und Lernen spielen im Future Skills Konzept eine konstitutive Rolle. Lernen wird als eigenaktiver Prozess verstanden, der in sozial-ökologischen Räumen stattfindet und durch diese gleichermaßen ermöglicht und begrenzt ist. Dabei wird Lernen auch an den Handlungsbegriff gekoppelt verstanden und als Aktivität aufgefasst, die dazu dient, subjektiv empfundene Handlungsbarrieren zu überwinden, eben durch Lernaktivitäten (Holzkamp 1993). Extern- bzw. fremdorganisiertem Lernen (etwa durch vorgegebene, nicht unmittelbar subjektiv relevante) Curricula können auch zu lernen führen, jedoch wird dieses mit Holzkamps (ebenda) subjektiver Lerntheorie eher als defensives Lernen verstanden.

Klaus Holzkamps subjektwissenschaftliche Grundlegung des Lernens (1993) geht von einer Analyse bisheriger lernpsychologischer Theorien aus und kommt dabei zu dem Schluss, dass das Subjekt in bisherigen Ansätzen nicht in ausreichendem Maße als selbstgesteuertes Individuum repräsentiert ist. Er analysiert die bisherigen (psychologischen) Herangehensweisen an das Konzept des Lernens als „Gleichsetzung mit fremdkontrolliertem "Lernen - und bezieht sich dabei auf behavioristische und kognitivistische Lerntheorien. So ist nach Holzkamp die Vorstellung, das Subjekt könnte ein Lebensinteresse am Lernen haben, in den von ihm analysierten Lerntheorien nicht zu finden. Das zu Grunde liegende Problem ist nach Holzkamp der „[...] Umstand, dass das Lernen als Problem vom wissenschaftlichen Standpunkt des Lernsubjekts in den traditionellen Lerntheorien nicht vorkommt" (Holzkamp 1993: 14). Es liegt nahe, dass hierin auch ein Grund dafür liegt, dass Theorien zur pädagogischen Qualität, die vom Lernsubjekt ausgehen, fehlen. 
Auf diese Analyse aufbauend schlüsselt Holzkamp das Lernen vom Subjekt her auf: Menschen erschließen sich demnach ihre Welt perspektivisch und mit intentionalem Bezug; die Wirklichkeit wird dabei vom Subjekt im Zusammenhang mit seinen Erfahrungen und Absichten gedeutet (vgl. Holzkamp 1993: 21). Das Subjekt stellt damit ein „Intentionalitätszentrum“ dar, „das von seinem Standpunkt aus auch andere Menschen als Intentionalitätszentren mit deren jeweils standpunktabhängiger Perspektive/ Intentionalität erfährt" (ebenda). Die Welt wird vom je eigenen Standpunkt aus als bedeutungsvoll wahrgenommen. Diese Bedeutungen werden zu Handlungsprämissen, auf deren Grundlage jeder Mensch aus für sich vernünftigen Gründen handelt (vgl. Holzkamp 1993: 26). Lernen wird als eine Art von Handlung dargestellt, die sich von anderen Handlungen durch das Ziel, die eigenen Verfügungsmöglichkeiten zu erweitern, unterscheidet.

Lernen ist ein für Bildung wichtiger und konstitutiver Prozess, der den Bildungsprozess unterstützen kann. Dabei wird Lernen nicht verstanden als hochschulisches Lernen im Sinne eines vorgegebenen Curriculums, sondern als Aktivität des sich bildenden Subjekts, die auch im Sinne von Sozialisation oder Entwicklung verstanden werden kann. Bei beiden Vorgängen kann auch von Lernen gesprochen werden, welches den Bildungsprozess befördert. Bildung in einem ganzheitlichen Sinne wird dabei verstanden als das Bemühen, ein dreifaches Verhältnis auszubilden, zu mir selber, zu einem Gegenstand und zur sozialen Umwelt. Es geht dabei darum, dass ich als mich Bildender ein Verhältnis zu mir selber ausbilde und insofern in eine kritische Distanz zu mir trete. Zweitens besteht es darin, dass ich in Bezug auf einen Gegenstand, oder ein Fach ein Verhältnis ausbilde, es mir beispielsweise aneigne, mich schlau mache, Wissen dazu erwerbe, mich qualifiziere. Drittens geht es um die Ausbildung eines Verhältnisses zwischen mir und der Umwelt, die sich oftmals auch als engere Umwelt, also andere Menschen und soziale System in die ich eingebunden bin darstellt. Oder die weitere Umwelt, meinen Arbeitsplatz, die Organisation in der ich arbeite oder die Gesellschaft. Keines dieser Pole steht für sich alleine oder kann gesondert betrachtet werden, denn meine Fähigkeit, in der jeweiligen Umwelt zu agieren wird wiederum durch meine Fähigkeiten in Bezug auf einen Wissensbestand oder bestimmte Fertigkeiten geformt, und auch dadurch, wie ich selber zu mir stehe, beispielsweise im Hinblick auf mein Selbstkonzept. Alle drei Pole stehen also in einem Verhältnis miteinander. Der so gefasste Bildungsbegriff liefert eine Struktur mit drei Polen, die in Verhältnissen untereinanderstehen. Diese Struktur entlässt uns jedoch nicht davon, uns die Pole, den Gegenstand, das Selbst/ die Persönlichkeit, und die Umwelt/ Gesellschaft selber anzusehen. Zwar beeinflussen sie sich alle gegenseitig im Bildungsverständnis und im Bildungsgeschehen, jedoch ist es hilfreich, sich die drei Pole und die Entwicklungen dort in den Blick zu nehmen. 
In den NextSkills Untersuchungen zu Future Skills und zur Frage, was an Wissensund Kompetenzbeständen bei Mitarbeiterinnen und Mitarbeiter in zukünftigen Arbeitsumfeldern gegeben sein muss werden in allen drei Polen des Bildungsbegriffes schematisch Wandlungstendenzen skizziert, die hier einmal aufgegriffen werden sollen. In Bezug auf die subjektive Dimension wird hervorgehoben, dass Selbstentwicklung, Autonomie und Reflexion wichtiger werden denn je, in Bezug auf die Objektdimension wird hervorgehoben, dass es einen Wandel vom objektiven Wissen hin zu sich schnell wandelnden Wissensbeständen gibt, so dass ein relativer Verfall - gewissermaßen inflationär - der Bedeutung dieser Dimension in ihrem Anteil an erfolgreiche Lösungen konstatiert wird. In Bezug auf die Umwelt/ soziale Dimension wird angemerkt, dass Organisationen sich im umfassenden Wandel innerer Organisation und Strukturbildung befinden, der durch umweltbedingte Megatrends ausgelöst (Demographischer Wandel, Digitalisierung, Globalisierung, Vernetzung) wird.

In Bezug auf die Frage, welche zukünftigen Kompetenzen nun wichtig erscheinen muss zunächst einmal festgehalten werden, dass sich die Struktur des so skizzierten Bildungsbegriffes nicht ändert, vielmehr die inhaltliche Ausprägung der drei Pole, die in der Struktur zusammenwirken. Die für die Bewältigung zukünftiger Aufgaben wichtigen Kompetenzen ergeben sich also im Verhältnis der sich vollziehenden Wandlungen. Zusätzlich tritt das Moment der Selbstorganisation hinzu, welches als grundlegende Anforderung und Bestimmungsgröße für kompetentes handeln Bedeutung hat.

\section{B 1.2.2 Der Kompetenzbegriff und Future Skills}

Welche Rolle spielt Kompetenz für Future Skills? Was ist der Zweck eines universitären Studiums? Bildung durch Wissenschaft oder Kompetenzentwicklung für den Beruf? Oder beides? Kompetenzorientierung ist zum Zauberwort für Lehren und Prüfen und damit auch für die Gestaltung von Studiengängen geworden. Dabei lässt sich Bildung sich nicht auf abprüfbare Kompetenzen reduzieren. Ein Hochschulstudium muss beides bieten: Möglichkeiten zum Erwerb von fachlichen und überfachlichen Kompetenzen, die sich als solche überprüfen lassen, und Gelegenheiten für Bildung durch Wissenschaft, die sich als Ganzes der Kontrolle weitgehend entzieht (Reinmann 2014).

Future Skills sind eine bestimmte Profilierung von bestehenden Kompetenzvorstellungen. Dabei gehen wir davon aus, dass Future Skills in besonderer Weise Kompetenzen beinhalten, die für zukünftige Handlungssituationen von Bedeutung sind. Was jemand dann dabei kann wird in Abhängigkeit von seiner persönlich 
emotional wertbezogenen Verfasstheit, vom jeweiligen Wissens- und Kenntnisstand und davon, wie er es in Bezug auf seine Umwelt einbringen kann bzw. wie seine Umwelt ihn in seinem Tun auch bereichern kann (siehe dazu auch Abbildung 11). Genau dieses Verständnis ist in dem Begriff der Kompetenz angelegt, wie ihn Erpenbeck et al. (2007) fasst. Im Mittelunkt steht dabei die Handlung, also das Agieren. Kompetenz als Konzept bezieht sich darauf, die Handlungsfähigkeit nicht nur in Abhängigkeit von Kenntnissen und Wissen zu sehen, sondern eben auch in Abhängigkeit der eigenen persönliche Werte, Einstellungen, Meinungen und Emotionen zu verstehen. Und drittens auf das Handlungssystem, in dem ich handele, also den Handlungskontext, in dem eine Handlung vollzogen werden soll, also der Handlungsumwelt. Kompetenz sind dabei nicht umwelt- oder kontextneutral, sondern beziehen sich immer auf einen bestimmten Kontext. Ein Beispiel ist die Kompetenz zu kommunizieren, die nicht kontextfrei verfügbar ist, sondern sich im Rahmen eines Geschäftsumfeldes anders gestalten kann als im Rahmen eines privaten Umfeldes. Nun kommt in Bezug auf Kompetenz noch eine weitere Dimension hinzu, die der Selbstorganisation.

\section{B 1.2.3 Das Konzept der Selbstorganisation}

Selbstorganisation ist ein Ankerkonzept für Future Skills. Selbstorganisation als Konzept ist von Heinrich Haken zum ersten Mal wissenschaftlich systematisch formuliert worden. Als Physiker bezieht er sich dabei auf die Fähigkeit von Teilchensystemen selbstständig Ordnungen und Strukturen zu bilden. Erpenbeck entwickelt diese Begrifflichkeit konsequent für das Feld der Kompetenzentwicklung weiter und benennt Selbstorganisation als eines der zentralen Merkmale von Kompetenz. Gewissermaßen ist Selbstorganisation damit eine unabdingbare Metadimension zu den drei genannten Dimensionen. Erst die Selbstorganisation als Metakategorie macht den Bildungsbergriff als Kompetenzkonzept fruchtbar.

Selbstorganisation ist gewissermaßen die vierte Dimension im dreifachen Strukturverhältnis des oben genannten Bildungsbegriffes. Das Monet der Selbstorganisation beeinflusst die jeweiligen Pole des Bildungsbegriffes und verändert so als Anforderung den Inhalt des Bildungsprozesses. Selbstorganisation in diesem Sinne kann nicht nur als Strukturbedingung eines jeden zukünftigen Bildungsvorganges verstanden werden, sondern auch als wichtiges normatives Element im Bildungsvorgang, welches die unterschiedlichen Bestandteile neu inhaltlich auflädt.

In Bezug auf Organisationen, Umwelt soziale Systeme bedeutet Selbstorganisation ein geringer werdender Einfluss hierarchisch, vorgegeben systemgrößten. In Bezug auf den Pol der Objekte von Bildungsbemühungen bedeutet Selbstorganisation als 
normative Orientierung, weniger vorgegebene kanonische Bildungsobjekte vorzusehen, und in Bezug auf die subjektive Dimension des strukturellen Bildungsbegriffes übersetzt sich Selbstorganisation in Selbstbestimmung und Autonomie und weniger fest bestimmte subjektive Verhaltens- und Lebensmuster.

Es sind die strukturellen Bedingungen, aus denen heraus die Kompetenzen definiert werden können, die Menschen zukünftig im Arbeits-, Privat- und Gesellschaftsleben in ihren Handlungen befähigen werden.

Darüber, was gelernt werden soll wird seit jeher gestritten. Unstrittig ist jedoch, der Entwurf einer Bildung zur Selbstbestimmung. Selbstbestimmung ist seit jeher ein wichtiges Ziel jeder Bildung in einer humanen, demokratischen Gesellschaft. Wenn man anerkennt, dass eines der allgemeinen Ziele einer humanen und demokratischen Erziehung - unter den Bedingungen unserer historischen Epoche - die Befähigung des jungen Menschen sein muss, in einem möglichst hohen Grade sich selbst bestimmen zu können, abgekürzt also Befähigung zur Selbstbestimmung, dann muss man zugleich Selbsttätigkeit als notwendiges pädagogisches Prinzip anerkennen (Klafki 2003). Dabei muss betont werden, dass Selbstbestimmung nicht subjektivistisch verstanden werden darf, sondern immer unter dem Gesichtspunkt der verantwortlichen Bezogenheit des einzelnen Menschen auf seine Mitmenschen, auf Kultur, Gesellschaft und Politik (ebenda).

\section{B 1.3 Stand der Dinge in der Future Skills Forschung}

Was sind die wesentlichen Ergebnisse bisheriger Forschungen zum Themenfeld Future Skills? Die Forschung zum Thema Future Skills gliedert sich in zwei verschiedene Bereiche: Zum einen in Forschungsarbeiten - Entwicklung von Rahmenkonzeptionen oder empirische Analysen von Anforderungen sowie Analysen von akademischen Curricula - zum Thema Graduate Attributes mit einem Höhepunkt in den 1990er Jahren. Zum anderen - gerade in jüngster Zeit, aufkommend seit den 2000er Jahren - zum Thema Future Skills oder 21st Century Skills. ${ }^{22}$ Ein weiterer, verwandter Forschungsbereich ist der Bereich der sogenannten Employability-Forschung,

22 Eine Recherche im Web of Science nach dem Begriff „21st century competences“ und „21st century skills“ führte zu folgendem Ergebnis: Drei akademische Veröffentlichungen für die Jahre 2000-2003, eine für die Jahre 2004-2007 und 19 für die Jahre 2008-2010, für die Jahre 2011-2014 158 und für die Jahre 2015-2019 299, allein Im Jahr 201939 Publikationen. Publikationen im Education Resource Center (ERIC) zeigen ein ähnliches Ergebnis: Für das Jahr 77 Publikationen, seit 2018143 Publikationen, seit 2015309 Publikationen, seit 468 Publikationen und seit 2000511 Publikationen. 
der seit den 2010er Jahren international boomt. Unsere Analyse des vorliegenden Forschungsstandes bezieht die meistzitierten Forschungsveröffentlichungen zum Thema Future Skills und Graduate Attributes aus den Jahren 2010-2019 mit ein. Zusätzlich haben wir mehr als 40 vorliegende Future Skills Konzeptionen analysiert, und auf deren inhaltliche Spannweite, sowie die genutzten Kategorien untersucht und vergleichend analysiert (siehe Kapitel B 1.4 Kritische Analyse bestehender Future Skills Konzeptionen). In breiten Linien lassen sich die Forschungsarbeiten der letzten 20 Jahre zu beiden Themen folgendermaßen zusammenfassen:

1. Forschung im Bereich der Graduate Attributes konzentriert sich darauf, zu ermitteln, welche Kompetenzen - als Attribute der Absolventinnen und Absolventen - besondere Relevanz besitzen bei deren späterem Erfolg auf dem Arbeitsmarkt. Abseits davon wird beforscht, welche Lehr-Lern-Strategien sich zur Entwicklung solcher Attribute besonders eignen, sowohl hochschuldidaktischer als auch curricularer Art. Trevleavan und Voola (2008) benennen nach ihrer Literaturdurchsicht elf verschiedene Begriffe für Graduate attributes: key skills, key competencies, transferable skills, graduate attributes, employability skills (Curtis \& McKenzie 2001), soft skills (BIHECC 2007; Freeman et al. 2008); graduate capabilities (Bowden et al. 2000); generic graduate attributes (Barrie \& Ginns 2004, Bowden et al. 2000); professional skills, personal transferable skills (Drummond et al. 1998); generic competencies (Tuning Report 2008). Rigby et al. (2009) fassen diese synonym verwendeten Begriffe unter dem Überbegriff der "graduate skills" zusammen. Sie beziehen sich damit auf solche Skills, die nicht nur für die berufliche Entwicklung relevant sind, sondern auch und vor allem die persönliche Entwicklung und die ganzheitliche Ausbildung des Individuums zu einem engagierten Mitglied der Gesellschaft fokussieren (ebenda: 4).

2. Employability, im Sinne von (lebenslanger) Beschäftigungsfähigkeit, lässt sich wissenschaftlich definieren und empirisch untersuchen. Dabei können Kompetenzen und Skills bestimmt werden, die für Employability Relevanz besitzen. (Forschung zeigt, dass Graduate Attributes wichtig für Employability sind)

a. In einer vergleichenden Literaturanalyse für die Jahre von 2006 bis 2014 sammelten beispielsweise Osmani und Kollegen (2015) ein 53 Graduate Attributes - umfassendes Set aus insgesamt 39 analysierten Studien.

b. Die australische Chamber of Commerce and Industry hat in Kooperation mit dem Business Council of Australia ein Set an Kompetenzen und personalen Attributen identifiziert, welche Arbeitnehmerinnen und Arbeitnehmer nach zu urteilen mit höheren Leistungen auf der Arbeit korrelierten (2000).

c. Der NCVER Report (2003) setzt Graduate Attributes zu Employability in Beziehung und kommt zu dem Schluss, dass Absolventinnen und Absolven- 
ten, die Graduate Attributes aufweisen, einen Wettbewerbsvorteil gegenüber Mitstreiterinnen und Mitstreitern mit schwach oder gering ausgeprägten Kompetenzniveaus im Hinblick auf Graduate Attributes haben.

3. Diejenigen Kompetenzen oder Skills, die für Employability besondere Relevanz besitzen sind oft überfachlicher Natur und werden als generische Kompetenzen, überfachliche Kompetenzen, Schlüsselkompetenz oder Soft-Skills bezeichnet.

a. Auf dem Arbeitsmarkt findet ein Wandel statt (Jackson 2014; James et al. 2004), der sich nun in den Curricula der Hochschulen niederschlagen sollte. Rigby et al. (2009) sprechen hierbei von einer notwendigen Verschiebung des curricularen Fokus weg von reinem Inhaltswissen, hin zu Prozesswissen. Dieser Wandel wirkt sich denn auch auf eine veränderte Pädagogik aus: Das Wissensvermittlungsparadigma soll um konstruktivistische Lehr-/Lernmodelle angereichert werden (Rigby et al. 2009: 5), was sich laut Tenenbaum et al. (2001) jedoch in der Praxis trotz curricularer Verankerung nicht notwendigerweise abbildet. Hauptursache hierfür mag vielfach die Unsicherheit des Lehrpersonals sein: Wer soll Graduate Attributes wie vermitteln und welche Methoden können zur Bewertung genutzt werden? (Freeman et al. 2008).

b. Wie Forschungsarbeiten belegen, müssen Absolventinnen und Absolventen nicht nur Graduate Attributes im Sinne von Fertigkeiten entwickeln, sondern ebenso die Bereitschaft und den Willen, diese in der Praxis anwenden zu können (Trevleavan \& Voola 2008; Hoban et al. 2004; Kember \& Leung 2005).

c. Rigby et al. (2009) zur Folge besteht das Kernproblem für eine Verankerung von Graduate Attributes in Hochschul-Curricula darin, dass bislang zwei widersetzliche Meinungen in der Literatur existieren, wie Graduate Attributes am besten vermittelt werden können: 1) Graduate Attributes zusammen mit fachlichen Kursinhalten schulen, wobei die zu trainierenden Graduate Attributes im jeweiligen Disziplinkontext relevant sein sollten (Barrie \& Ginns 2004; Sin \& Reid 2005; Thompson et al. 2008; Bowden et al. 2000; Star \& Hammer 2007; Drummond et al. 1998; Bath et al. 2004). 2) Graduate Attributes Disziplinen - unabhängig in separaten Kursformaten vermitteln (Cranmer 2006). Während der erste Ansatz davon ausgeht, dass sich Lehrformen zugunsten geänderter Nachfragen des Marktes ändern müssen (Biggs 2003), sucht letzterer in Form eines Baukasten-Prinzips Skill-Defizite einzelner Studierender nachzurüsten, ohne dabei die Notwendigkeit in veränderten Lehrkonzeptionen zu fokussieren. Osmani et al. (2015) schlagen einen „Dopplungsansatz" vor, welcher vorsieht, Graduate Attributes einerseits im Curriculum zu verankern (1), andererseits und darüber hinaus zusätzliche Employability-Programme und oder Workshops anzubieten. 
4. Es kann festgestellt werden, dass generell ein Defizit bei den Curricula der Hochschulen dahingehend besteht, diese auf die Förderung von besonders employability-relevanten Kompetenzen auszurichten.

a. Finch, Hamilton, Baldwin und Zehner (2013) identifizierten in ihrer Studie Faktoren, welche einen Einfluss auf die Employability von Absolventinnen und Absolventen haben, wobei sich zeigte, dass Arbeitgeber den sogenannten Soft Skills am meisten Bedeutung zumaßen; akademische Reputation wurde als am unbedeutendsten eingestuft. Ähnliches findet sich auch in den Studienergebnissen von beispielsweise Daud et al. (2011) oder Finch et al. (2013).

b. Im Report zur Zufriedenheit von Arbeitgebern mit dem Level von Graduate Attributes bei ihren Angestellten, zeigen Hager et al. (2002) auf, dass die Leistungen der Mitarbeiterinnen und Mitarbeiter nur als ,,angemessen“ evaluiert wurden. Dies ist als Fingerzeig in Richtung der Hochschulen aufzufassen, welche es bislang versäumt haben, die für den Markt kritischen Kompetenzen in angemessener Weise bei ihren Absolventen zu schulen.

c. Rigby et al. sprechen in diesem Zusammenhang auch von einem ,implementation gap“ (2009: 8), Osmani et al. (2015) betiteln diese als „broad mismatch“ (siehe ebenda 367).

d. Tran (2015) zufolge sind Hochschulabsolventen schlecht auf den Arbeitsmarkt und dessen Anforderungen vorbereitet, da Curricula häufig veraltet oder irrelevant seien.

e. Studienergebnisse von Gibbs et al (2011) sowie Stone, Lightbody und Whait (2013) legen nahe, dass Kooperation und Dialog zwischen Stakeholdern (Hochschulen, Arbeitgeber, Studierende, ...) der Schlüssel dafür ist, Skill-Bedarfe und -Schulungsangebote adäquat auszuloten und miteinander in Einklang zu bringen. Zu diesem Schluss kommen auch Daud et al. (2011), die in ihrer Studie eine Lücke zwischen den von Arbeitgeberseite geforderten Graduate Attributes der Absolventen eines Business- und Managementstudiums und der Performance dieser Absolventen nach ihrem Studium aufdeckten. Daher sollte - so der Schluss der Autoren - Curriculum-Design immer die Perspektive des Arbeitnehmers mitberücksichtigen und die Frage danach stellen, welche Kompetenzen künftige Absolventinnen und Absolventen in ihrem späteren Arbeitsfeld benötigen.

f. Dewey und Kollegen (2008) analysierten die Erwartungslücke zwischen Kompetenzen, die Absolventinnen und Absolventen eines Graduiertenkollegs nach ihrem Studium aufwiesen und verglichen sie mit denjenigen Kompetenzen, die von den Arbeitgebern als wichtig erachtet wurden. Es zeigte sich, dass es Diskrepanzen zwischen der Erwartungshaltung der Arbeitnehmerinnen 
und Arbeitnehmern und den im Ausbildungsprogramm vermittelten Kompetenzen der Schule gab.

g. In der US-Literatur wird die Lücke zwischen den durch die Industrie nachgefragten und den an Hochschulen gelehrten Skills durch eine Reihe von empirischen Studien belegt (z. B. Aasheim, Williams \& Butler (2009); Cox et al. (2013); Koppi et al. (2009); Koppi et al. (2009)). Koppi und Kollegen (2009) untersuchten beispielweise, wie das Curriculum von US-Bachelor-Studierenden besser an die Anforderungen des Arbeitsmarktes angepasst werden könnte. Dabei zeigte sich, dass nicht die Aufteilung von Wirtschafts- und Technik-Kursen einer Adjustierung bedurfte, sondern dass das Curriculum stattdessen verstärkt darauf ausgerichtet werden sollte, Kommunikationsund Teamarbeits-Skills in den Fokus zu stellen.

5. 21st Century oder Future Skills sind ein erst in jüngerer Zeit durch das World-Economic Forum, die UNESCO, die Europäische Kommission oder die OECD aufgekommener Forschungsgegenstand, der sich mit der Frage beschäftigt, welche Graduate Attributes besondere Relevanz besitzen, um in einer sich zunehmend globalisierten und digitalisierten Welt gesellschaftlich gestalterisch, verantwortungsvoll, nachhaltig und im Sinne der Millennium-, bzw. Sustainable Development Goals zu agieren (Osmani et al. 2015; Rigby et al. 2009).

Die Einbettung und Integration effektiver Skill Entwicklung wird trotz der bereits Jahre andauernden Diskussion und Forschung immer noch als „difficult to operationalize effectively“ (Drummond, Nixon, \& Wilkshire (1998: 21) bewertet.

6. Die aus den letzten 10 Jahren vorliegenden Ansätze zu 21st century skills und aus den letzten 5 Jahren vorliegenden Ansätze zu Future Skills sind vielfach orientiert an der Gestaltung von politischen Rahmenempfehlungen, und sind nicht immer empirisch fundiert oder basieren lediglich auf einer sektoralen Datenerhebung. Daher besitzen Studien, wie die vorliegende, die Future Skills empirisch operationalisieren besondere Relevanz, um diese Lücke zu schließen.

7. Die vorliegenden Ansätze bestehen in der Regel aus Listen von mehr oder weniger wichtigen Fähigkeiten, jedoch basieren die Ansätze nicht auf fundierten kompetenztheoretischen Ansätzen (Barrie 2004; Clanchy \& Ballard 1995; Sin \& Reid 2005). Es findet keine Modellbildung statt, die ermöglicht, die Modelle hinsichtlich ihrer Substanz und Reichweite kritisch einzuordnen.

8. Bei den meisten der vorliegenden Ansätze wird deutlich, dass sie weit darüber hinaus gehen, aufzulisten, was Absolventinnen und Absolventen wissen sollten (Wissen) und zu tun in der Lage sein sollten (Fähigkeiten) und sich darüber hinaus auf einen großen Bereich von persönlichen Charakteristika beziehen (Rigby et al. 2009). Rigby et al. (2009) fassen daher unter Graduate Attributes 
nicht nur einzelne Skill Komponenten, sondern auch Einstellungen, Werte, Dispositionen, Fähigkeiten und Kompetenzen von Individuen.

9. Ein interessanter Ansatz ist dabei, Attribute und Skills für Employability nicht als Listen von Eigenschaften und Fähigkeiten zu verstehen, sondern in einem weiteren Sinne als Teil der Identität zu fassen, die im Rahmen von akademischen Studien ganzheitlich entwickelt werden soll. Diese Ansätze beziehen sich vor allem auf Bourdieu (z. B. 1986 1990) und beziehen Habitus (Internalisierung von kulturellen Normen) und Kapital (soziales, kulturelles und ökonomisches Kapital) als Komponenten mit ein. Diese Ansätze fokussieren nicht auf die Aneignung von einzelnen aufzulistenden Skills, sondern vielmehr darauf, Studierende bei der Transformation in ihre professionelle Rolle im Berufsleben zu begleiten. Diese mehr ganzheitlichen Ansätze erscheinen vielversprechend, sind aber noch selten. Osmani und Kollegen (2015) sprechen sich deshalb auch dafür aus, Graduate Attributes in die hochschulischen Curricula mit aufzunehmen, um den Anforderungen der Arbeitswelt von morgen bestmöglich begegnen zu können.

\section{B 1.4 Kritische Analyse bestehender Future Skills Konzeptionen}

Welche Future Skills Modelle und Konzeptionen gibt es derzeit und wie sind diese aufgebaut? Eine Recherche zu den derzeit verfügbaren Future Skills Ansätzen, Modellen und Konzeptionen kann nur unvollständig bleiben. Zu dynamisch ist dieser Bereich und zu divers die Verständnisse davon, was zu Future Skills dazu gehört, was vielleicht als 21st Century bezeichnet wird, aber eigentlich Future Skills meint oder was sich auf bestimmte Bildungssektoren - etwa Schule, Lehrerbildung, Hochschule, einzelne Hochschuldisziplinen, etwa dem Ingenieurwesen (beispielsweise Der Ingenieur/ die Ingenieurin 4.0) oder Wirtschaft (beispielsweise Leadership Skills für Managerinnen und Manager) - oder inhaltliche Domänen, wie beispielsweise MINT/ STEM Skills bezieht. ${ }^{23}$ Ein inhaltlich-analytischer Vergleich der Ansätze ist aufgrund dieser Heterogenität nicht sinnvoll. Die Ansätze können jedoch anhand eines einheitlichen Kriterienrasters von Skills nebeneinander vergleichend dargestellt werden, um einen Eindruck von Umfang und Abdeckung der jeweiligen Ansätze zu bekommen. Um diese Kriterienraster zu erhalten, wurde eine Metanalyse durchgeführt.

23 STEM kommt aus dem Englischen und bedeutet Science, Technology, Engineering und Mathematics. Vergleichbar zum engl. STEM ist das deutsche Akronym MINT, welche sich auf Mathematik, Informatik, Naturwissenschaft und Technik bezieht. 
Tab. 2 Vergleichende Analyse bestehender Future Skills Modelle (Quellen siehe Literaturverzeichnis)

\begin{tabular}{|c|c|c|c|c|c|c|c|c|c|c|c|c|c|c|c|c|c|c|}
\hline $\begin{array}{l}\text { Consolidated } \\
\text { Skill Inventory }\end{array}$ & 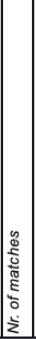 & 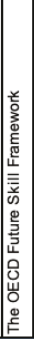 & 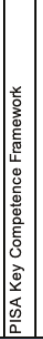 & 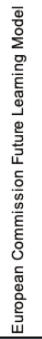 & 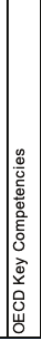 & 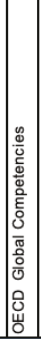 & 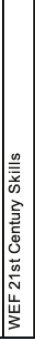 & 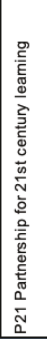 & 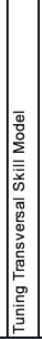 & 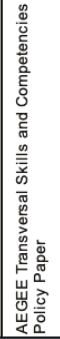 & 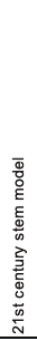 & 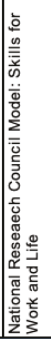 & 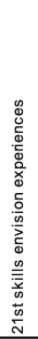 & 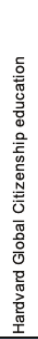 & 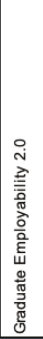 & 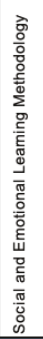 & 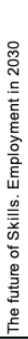 & 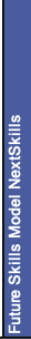 \\
\hline \multicolumn{19}{|c|}{ Subject oriented skills } \\
\hline \begin{tabular}{|l} 
Analytical and critical thinking \\
\end{tabular} & 10 & & & & & 1 & 1 & 1 & 1 & & 1 & 1 & 1 & & & 1 & 1 & 1 \\
\hline \begin{tabular}{|l} 
Creativity \\
\end{tabular} & 11 & 1 & 1 & 1 & & & 1 & 1 & 1 & & & & 1 & & 1 & 1 & 1 & 1 \\
\hline Learning skills & 6 & & & & & 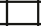 & & & 1 & 1 & 1 & 1 & & & & & 1 & 1 \\
\hline Action \& Initiative & 6 & 1 & & 1 & & & & 1 & & 1 & & & & & & 1 & & 1 \\
\hline Health Literacy & 4 & & 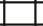 & & & 1 & 1 & & & & & & & & & 1 & & 1 \\
\hline $\begin{array}{l}\text { Intercultural knowledge and } \\
\text { understanding }\end{array}$ & 9 & & 1 & & 1 & & 1 & 1 & 1 & 1 & & & 1 & & & 1 & & 1 \\
\hline \begin{tabular}{|l} 
Taking Responsibility \\
\end{tabular} & 5 & 1 & & 1 & & 1 & & 1 & & & & & & & & & & 1 \\
\hline Persistence/grit & 4 & & & & & 1 & 1 & & & & & & & & & 1 & & 1 \\
\hline Ability to reflect & 3 & 1 & & & & & & & & 1 & & & & & & & & 1 \\
\hline Curiosity & 3 & & & & & 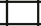 & 1 & & & & & & & & & 1 & & 1 \\
\hline Entrepreneurship Skills & 5 & & & & 1 & - & & & 1 & 1 & & 1 & & & & & 1 & \\
\hline Flexibility & 3 & & & & & 1 & & 1 & & & & & 1 & & & & & \\
\hline Global-mindedness & 2 & & & & 1 & 1 & & & & & & & & & & & & \\
\hline Anticipation & 2 & 1 & & & & & & & & 1 & & & & & & & & \\
\hline Deal w. ambiguity and uncertainty & 2 & & & 1 & & & & & & & & & & & & & & 1 \\
\hline Empathy & 2 & & & 1 & & 1 & & & & & & & & & & & & \\
\hline $\begin{array}{l}\text { Form \& conduct life plans, personal } \\
\text { projects }\end{array}$ & 2 & & 1 & & & & & & & 1 & & & & & & & & \\
\hline Resilience & 2 & & & 1 & & & & & 1 & & & & & & & & & \\
\hline Compassion & 1 & & & 1 & & & & & & & & & & & & & & \\
\hline Failing Forward & 1 & & & 1 & & & & & & & & & & & & & & \\
\hline Reconciling Tensions \& Dilemmas & 1 & 1 & & & & & & & & & & & & & & & & \\
\hline Risk Taking & 1 & & & 1 & & & & & & & & & & & & & & \\
\hline \multicolumn{19}{|c|}{ Object related skills } \\
\hline Digital \& Data Literacy & 9 & 1 & & & 1 & 1 & 1 & 1 & & & & 1 & 1 & & & 1 & & 1 \\
\hline STEM skills, complex problem solving & 7 & & & & 1 & & 1 & 1 & & & 1 & 1 & 1 & & & & 1 & \\
\hline Financial Literacy & 5 & & & & & 1 & 1 & 1 & & 1 & & & & & & 1 & & \\
\hline \multicolumn{19}{|c|}{ Organisation related skills } \\
\hline $\begin{array}{l}\text { Communication skills (language, } \\
\text { symbols, texts) }\end{array}$ & 14 & & 1 & & 1 & 1 & 1 & 1 & 1 & 1 & 1 & 1 & 1 & 1 & 1 & & 1 & 1 \\
\hline teamwork & 8 & & 1 & 1 & 1 & & & & 1 & & & & 1 & 1 & & 1 & & 1 \\
\hline Context awareness and adaptability & 5 & & & & & 1 & 1 & 1 & & & & & 1 & & & 1 & & \\
\hline Leadership skills & 8 & & & & & 1 & 1 & & 1 & & 1 & 1 & & 1 & 1 & 1 & & \\
\hline Co-operation skills & 9 & & 1 & & & & 1 & & 1 & & 1 & 1 & 1 & 1 & & & 1 & 1 \\
\hline Networking skills & 7 & & 1 & 1 & 1 & & & & & 1 & & & & 1 & 1 & 1 & & \\
\hline $\begin{array}{l}\text { Interact respectfully, appropriately and } \\
\text { effectively }\end{array}$ & 5 & & & & & 1 & & & & 1 & 1 & 1 & 1 & & & & & \\
\hline Civic Competence & 4 & & & & 1 & & & & & 1 & & & & & & 1 & & 1 \\
\hline Co-constructing, shared cognition & 2 & & & 1 & & & & & & & & & & & & & & 1 \\
\hline Act within the big picture & 1 & & 1 & & & & & & & & & & & & & & & \\
\hline $\begin{array}{l}\text { Defend and assert rights, interests, } \\
\text { limits and needs }\end{array}$ & 1 & & 1 & & & & & & & & & & & & & & & \\
\hline Organizational Skills & 4 & & & 1 & 1 & & & & & & 1 & 1 & & & & & & \\
\hline Tot & & & & 13 & 10 & & 13 & 11 & 10 & 12 & 8 & 10 & 11 & 5 & 4 & 14 & & \\
\hline
\end{tabular}


Dazu wurde folgendermaßen vorgegangen: Unter Zuhilfenahme von den Schlagwörtern „Future Skills “, „21st Century Skill“, „Future Learning“, „Future Higher Education“ konnten zunächst 41 Modelle, Ansätze, politische Positionspapiere und Konzeptionen ermittelt werden, die innerhalb des Zeitraums zwischen 2012 und 2019 publiziert wurden. In die Analyse wurden dabei ausschließlich explizite und konkrete Konzeptionen einbezogen, die Skill-Beschreibungen und Listen von Fähigkeiten enthielten. Rein theoretische Konzeptionen wurden in dieser Analyse nicht berücksichtigt. Sie sind Gegenstand der Analyse in Kapitel B 1.3.

Im nächsten Schritt wurde eine Longlist erstellt, die alle Skill Items aller 41 Skill Ansätze enthielt. Sie resultierte in insgesamt 199 Items. Diese wurden mittels einer inhaltsanalytischen Vorgehensweise durch Paraphrasierung sowie Ermittlung und Vereinheitlichung von Doppelnennungen harmonisiert. Dadurch konnten die 199 Items auf 33 Items reduziert werden, die in Formulierungstiefe und Konzeptbreite geeignet waren, als Kategorienraster bzw. Vergleichskriterien für die zuvor ermittelte Gesamtliste der 199 Items zu fungieren. Die 33 Vergleichskriterien wurden dann in einem weiteren Schritt inhaltlich in die drei Kategorien eingeteilt, die im Triple Helix-Modell für Future Skills konstruiert wurden - also Sills, die sich auf subjektiv-individuelle Fähigkeiten, wie beispielsweise Reflexionsfähigkeit beziehen, solche, die sich auf Gegenstände, Objekte bzw. inhaltliche Expertisebereiche beziehen, wie beispielsweise STEM Kompetenzen (objektbezogene Kompetenzen), und solche, die sich auf Kompetenzen im Umgang mit der sozialen Umwelt beziehen, hier als organisationsbezogene Kompetenzen bezeichnet (siehe Tabelle 2).

Im nächsten Analyseschritt wurden wurde 17 aus den 41 Skill Ansätzen ausgewählt, die in die vergleichende Darstellung mit einbezogen werden sollten. Dabei wurden aus den zuvor zugrunde gelegten Skill Konzeptionen, Ansätzen und Modellen solche Ansätze mit einbezogen, die explizit Future Skill Listen enthielten. Diese wurden dann anhand der 33 Kriterien miteinander vergleichend dargestellt. Das Ergebnis ist in Tabelle 2 dargestellt. Die Future Skills, die am häufigsten in den miteinander verglichenen Ansätzen zu sehen sind, sind - mit jeweils mehr als 5 Nennungen - folgende Skills:

- Creativity

- Analytical and critical thinking

- Intercultural knowledge and understanding

- Learning skills

- Action \& Initative

- Taking Responsibilty

- Digital \& Data Literacy

- STEM skills, complex problem solving 
- Communication skills (language, symbols, texts)

- Co-operation skills

- Teamwork

- Leadership skills

- Networking skills

- Context awareness and adaptibility

- Ability to interact appropriately and effectively 